\title{
STORAGE OF NORMAL TISSUE GRAFTS
}

A

SYMPOSIUM, under the chairmanship of Dr. A. S. Parkes, on the "Preservation of Normal Tissues for Transplantation" was held in the rooms of the Ciba Foundation during March 16-18, 1953. Like other colloquia organized by the Foundation, its purpose was to provide not merely for the formal presentation of scientific papers but also for the informal discussion of problems of common interest to the participants. The symposium kept closely to the subject defined by the title; tumour transplantation was left aside because tumours are 'abnormal', and the storage of semen because semen is not a tissue.

Tissue storage, for practical medical purposes, is a storage of spare parts for surgical replacement. It is possible to envisage situations in which a patient's tissues might be stored for his own future use-for example, a surgeon might deliberately remove a little more skin than was necessary at an orthodox grafting operation, and keep the excess in store in case some of the grafts he actually used failed to heal properly - but for nearly all practical purposes, storage for replacement surgery entails the use of tissues from other human beings. This being so, the surgical use of stored tissues is bedevilled by the fact that some living tissues will not form permanent grafts after transplantation from one individual to another. They elicit immunity reactions which end by destroying them. Problems of tissue storage are thus closely interwoven with problems of tissue transplantation immunity.

In opening the scientific proceedings, Prof. P. B. Medawar (University College London) said that living skin, when transplanted into positions formerly occupied by skin, was probably the most exacting of all tissues in this regard; skin so grafted between one adult and another did not survive longer than a few weeks. Other tissues enjoyed special dispensations from the rigours of transplantation immunity, the nature of which could only be understood if the normal process of immunity were first defined. 'There is no doubt at all that intolerance of homografts had the properties of an actively acquired immunity reaction, but a reaction of what particular kind? A certain combination of properties points unmistakably to membership of that class of immunity reactions of which tuberculin sensitivity and sensitization to simple chemical compounds were also members. The most recently discovered of these properties, so Mitchison had found, was that immunity could be passively transferred by living cells but not by serum.

A homograft could be made more acceptable to its host either by changing the antigenic propensities of the graft or by modifying the reaction of its host. Under the latter heading, Prof. Medawar outlined experiments done in collaboration with $R$. $E$. Billingham and L. Brent which showed that if an animal were presented with living foreign cells in foetal life, its power to react against those cells in later life was reduced or wholly abolished. This was not due, as had been widely assumed, to an adaptation of the grafted cells, but to an adaptation of the host, for 'actively acquired tolerance', once established by inoculation of the fotus, extended to cells freshly transplanted in later life--cells which therefore had had no opportunity to adapt themselves to alien soil.
Dr. W. P. Longmire (University of California and U.S.A.A.F.) proceeded to survey the clinical uses of tissue homografts in general. One could make a broad distinction between homografts which had to be alive if they were to serve a permanently useful purpose, and homografts which could act as a guide and sometimes a stimulus to natural regeneration irrespective of whether they remained alive or not. Skin and endocrine glands came pre-eminently into the former category, of 'homovital' grafts. 'Homostatic' grafts included those of artery, bone, cartilage and possibly cornea. Grafts of some of these tissues did not even have to be alive on transplantation, but corneal grafts which were dead on transplantation had not given successful results. Dr. Longmire mentioned that plastic substitutes behaved differently from homostatic grafts : they might be tolerated by, but were not incorporated into, the body. Turning to experiments on fundamental problems, Dr. Longmire said that he and his colleagues were engaged with studies on the success of skin transplantation in chicks as a function of age. A small proportion of skin homografts were successful when exchanged between newly hatched chicks of different breeds; but the proportion of successes fell off rapidly to zero by the time the chicks were two weeks old. By holding the age of the recipient constant, it had been found that younger skin had somewhat less power to provoke immunity than older skin.

Dr. J. W. Pate (U.S. Naval Medical Centre) adopted Dr. Longmire's classification of homografts, and said that, inasmuch as certain homografts did not have to be alive on transplantation, their storage in the living state raised unnecessary complications. Some storage treatments entailed chemical modification of the grafted tissue ; generally, storage by freezing or freeze-drying was therefore to be preferred. Experiments showed that vitality of arterial homografts was not merely unnecessary but undesirable: frozen-dried arterial homografts were superior to fresh living homografts, and frozen-dried bone was not inferior to living bone. Frozen-dried skin could be used as a homostatic graft for providing a temporary physiological dressing for raw areas on severely burnt patients, in full recognition of the fact that they contained no living cells. Frozen-dried skin was as useful as a living skin homograft for such a purpose.

Dr. W. R. Earle (National Institutes of Health, Bethesda) described his methods for cultivating tissues in vitro in large quantities and for long periods. Such mass cultures could be started from single cells grown in capillary tubes, and might be subdivided into sublines which proved to have different properties; nor was growth confined to 'two-dimensional' sheets, for the incorporation of glass helices into the medium provided a framework for solid growth. Dr. Farle had cultivated human skin for $1 \frac{1}{2}$ years in vitro, and one such culture attained a total area of $1,600 \mathrm{~cm},{ }^{2}$; he emphasized the tremendous possibilities for research which such materials and techniques now placed at the investigator's disposal.

The second session of the conference was concerned with the problem of storing and grafting endocrine tissue. Dr. Parkes (National Institute for Medical Research) began with a paper by Dr. Audrey Smith 
and himself on ovarian grafts. Early experimenters, he said, had approached the problem of endocrine homografts with complete disregard of immunological complications, and whether this attitude were wholly justifiable or not, he himself was convinced that endocrine homografts were both practicable and effective. Ovarian tissues impregnated with 15 per cent glycerol in normal saline and slowly frozen to - $190^{\circ} \mathrm{C}$. did not deteriorate after a year's storage ; when thawed and transplanted into ovariectomized recipients, vaginal œstrous cycles were restored. Rat testes, and probably the pituitary, could be stored in the same way, but the adrenal gland had proved more difficult.

This work was concerned purely with the endocrine activity of stored ovarian tissue ; Dr. R. Deanesley (National Institute for Medical Research) considered the degree to which it retained its gametogenic power. Small pieces of ovary from 7-day old rats were frozen and later grafted into older recipients ; nearly all the ova had disappeared twenty-four hours later, but healthy follicles could be identified in specimens removed for microscopic examination after 6-10 days. These follicles increased in size with age, and became prematurely luteinized. Similar experiments with frozen testis material showed that it, too, survived such treatment : it remained capable of maintaining histological structure of the seminal vesicles of castrated hosts and gave evidence even of sperm formation.

Dr. P. J. Gaillard (Leyden) recapitulated the results of earlier experiments of his which showed that fœtal tissues could acquire differentiated functional activity if cultivated in media derived from older animals. These experiments opened up the possibility of using endocrine grafts originally derived from fœtuses. Human fœtal parathyroid glands had been maintained in culture for seven weeks, the last stages of cultivation being in media derived from their prospective recipients. The cultivated tissues were transplanted into perivascular tissues of patients who had shown signs of parathyroid deficiency after thyroidectomy. In a number of such patients the calcium and phosphorus levels in the blood were rapidly restored to the normal. Success was more common in the younger patients; all the grafts had failed in patients more than thirty-six years old. Dr. P. F. Jones reported that homografts of adrenal cortical tissue from rats, not more than two days old, could survive in mature adrenalectomized rats of a different strain and could keep their hosts alive for many months. The grafts were well vascularized and looked healthy; their hosts died shortly after the removal of the grafts, so showing that the cortical tissue was actively secreting adequate amounts of hormones.

The third session began with a consideration of the storage of blood. The preservation of even the most labile plasma components, Dr. P. L. Mollison (Postgraduate Medical School) said, was relatively simple. Transfused leucocytes soon left the circulation, so that the principal problem was the preservation of red cells. Red cells can be identified by their antigenic properties; their average life-span was about 115 days. Storage in citrated glucose at $4^{\circ} \mathrm{C}$. gave poor results after about a month; maintenance of a $p H$ of 7.2 improved the results, and sucrose could be used to delay hæmolysis without, however, improving the survival of red cells after transfusion. Dr. Mollison particularly emphasized the fact that the non-occurrence of hæmolysis or visible abnormality after storage did not by any means guarantee the prolonged survival of red cells after transfusion.

The incorporation of glycerol into storage media made possible the use of storage temperatures far below zero, and therefore storage for long periods; but great practical difficulties were introduced by the need for removing glycerol slowly from red cells stored in bulk. Although there was always some loss of cells on storage at very low temperatures, the deterioration did not increase as storage was prolonged. Dr. Mollison said that transfused red cells sometimes failed to survive, and he wondered whether a reaction akin to that provokod by living nucleated cells could be responsible.

The best rates for freezing and thawing tissues to ensure their survival, and the remarkable protective powers of glycerol, were all discovered and worked out by pure empiricism, sometimes (as with the choice of slow freezing in preference to fast) in the face of expert theoretical advice to the contrary. Dr. J. E. Lovelock (National Institute for Medical Research) considered the biophysical aspects of the process of freezing and showed to what a large extent a reconsideration of the theoretical background of the process had justified the empirical choice of methods. The effect of glycerol was to reduce the range of temperature over which cells were particularly susceptible to damage during freezing ; its efficacy depended among other things on its complete non-toxicity, its low molecular weight and free passage across cell membranes; very few other compounds, if any, had just the right combination of properties.

Dr. C. M. Pomerat (University of Texas) had been engaged in an attempt to interpret the behaviour of tissues in bulk during changes of temperature into the responses of individual cells. Apparatus had been devised which made it possible to combine continuous thermal and photographic recordings of cultivated cells-particularly those of human nasal mucosaunder various conditions of thermal stress. The forms of mitochondria and the vigour of intracellular movement were sensitive indicators of a cell's wellbeing under such conditions. Describing the effects of glycerol, Dr. Pomerat illustrated the vigorous cytoplasmic bubbling consequent upon a rapid transition from glycerol to normal saline solutions. Pinocytosis and nuclear rotation were also illustrated; further study would probably show that these also served as delicate indicators of departures from normality of behaviour.

Dr. R. E. Billingham (University College London) began the fourth session by describing the storage of skin. After impregnation with glycerol solutions, skin slowly frozen to $-79^{\circ} \mathrm{C}$. did not deteriorate in any way after even ten-months storage; used as a graft, it was indistinguishable from an unstored normal skin graft in fine structure, colour, epithelial growth-rate, and differentiation of glands and hairs. Such eriteria of normality were much more rigorous than those based on mere cellular survival. Melanocytes were much more exacting than Malpighian cells in their storage requirements, and showed up the advantages of using glycerol particularly well. Skin did not survive desiccation from the frozen state below a final overall water content of about 25 per cent; claims that mammalian tissues could withstand a higher degree of dehydration had never been supported by actual estimates of residual water. Glycerol, Dr. Billingham pointed out, was negligibly toxic; even dissociated epidermal cells could with- 
stand being suspended in 98.1 per cent glycerol at room temperature for half an hour.

Dr. F. K. Sanders (University of Oxford) described experiments on the use of nerve homografts. Frozendried homografts were capable of guiding regenerating nerve fibres across relatively large gaps in the nerve trunks of small laboratory animals, their endoneural collagenous tubes being systematically re-populated by Schwann cells and fibroblast cells deriving from the host. Fresh homografts elicited a typical homograft immunity reaction in which the vascular stagnation was particularly inimical to successful repair. Results with the use of nerve homografts in human beings had been particularly disappointing. Dr. Sanders argued that this was because the absolute size of such gaps was always larger in clinical practice than in experimental animals; since the time relations of the immunity reaction were not much different in the two cases, and the rate of outgrowth of fibres from the central stump much the same, the difference was probably due to the fact that in human beings the immunity reaction became effective before the regenerating fibres reached the peripheral stump.

The next two papers were concerned with the use of arterial homografts, among the most uniformly successful and rewarding of all those in clinical use. Prof. G. C. Rob and Dr. H. H. G. Eastcott, of St. Mary's Hospital, used normal human vessels, removed as quickly as possible after death and stored in the frozen state. Such grafts gave excellent functional results, the success of which depended neither upon the viability of the graft at transplantation nor upon its length ; the new endothelial lining was formed as quickly in long grafts as in short, and they accordingly inferred that it was formed by cells derived from the blood stream and not by creeping invasion from the two ends of the graft. Dr. C. A. Hufnagel (Georgetown University) summarized several years experience with vascular grafts in clinical practice and in experiments with dogs. There was no elinical distinction between the success of fresh, frozen, or frozen-dried grafts, and even frozen-dried heterografts from calves and pigs had given clinically satisfactory results. But even frozen-dried grafts had to be removed and preserved aseptically; experiments still in progress suggested that ethylene dioxide was an efficient sterilizing agent which produced no significant alteration in the fine structure of the grafts.

In clinical practice, transplantation of the cornea must always be of necessity homoplastic, and it is now quite widely known to enjoy an encouragingly high expectation of success. Mr. B. W. Rycroft (East Grinstead) analysed the criteria of success. Mere healing, and even the restoration of translucency, were not enough: there must be improvement of vision, if the graft were done for other than purely cosmetic purposes or to prepare the cornea for a later definitive grafting. Lamellar grafts were safer and generally more successful than full-thickness grafts, in which the danger of infection became serious; but he believed that the optical virtues of the latter might be combined with the safety of the former by means of a 'flanged' graft, or full-thickness centrally but with a thinned rim. Mr. Rycroft believed that corneal grafts were replaced by the tissues of the host ; but heterografts were generally unsuccessful, and plastic substitutes inefficacious. One of the chief practical difficulties was over supply. The Corneal Grafting Bill of 1952 had now at last made it possible for eyes to be bequeathed after death, and such eyes might be stored for perhaps two weeks under liquid paraffin; but a method of semi-permanent storage would clearly be of the greatest value.

The proceedings ended with Dr. W. R. Strong's (U.S. Naval Medical Centre) description of the working of the U.S. Naval Tissue Bank, instituted in 1949. The Bank made use of tissues aseptically removed after death and stored after desiccation from the frozen state to less than 1 per cent residual water. Skin, bone and blood-vessels were systematically removed in accordance with an exacting routine and stored after drying in sealed glass vessels. Frozendried bone, even in the form of coarse granules, had given encouraging clinical results, and so, in their more limited experience, had blood-vessels. Frozendried skin was used purely as a temporary physiological dressing : it relieved pain, controlled the loss of tissue fluids, and reduced the risk of infection.

The impressions left in the mind of one participant were these. 'Homovital' grafts (homografts which must be alive on transplantation and must remain so if they are to serve a useful purpose) are a welldefined category. The use of skin homografts in clinical practice must await a fuller analysis of the methods by which their putative recipients can be made to tolerate them. Such methods have already been discovered; but they are not yet clinically applicable, and it is not likely that anything but fundamental research will make them so. Endocrine homografts sometimes succeed, and treatment of endocrine deficiency by hormones has not yet stolen all the thunder their use might one day cause. At the same time, it would be unfortunate if the dispensation which allows them sometimes to survive where homografts of other tissues fail were not made the subject of thorough scientific analysis; the theory of endocrine homografting as such can at present only be pieced together most incompletely from research conducted with only endocrinological or clinical purposes in mind. Tissue storage will become of practical value when the difficulties of transplantation have been overcome. The storage of skin offers no particular difficulty; nor that of certain endocrine tissues -at least if ambitions fall short of storage in perpetuity. The different endocrine tissues may raise their own peculiar storage difficulties.

'Homostatic' grafts are a less well-defined category. Some must apparently be alive on transplantation, but need not remain so ; others may be dead from the beginning; some, perhaps all, provide stimuli for regeneration which could not be imparted by plastic replacements; certainly all require that a certain integrity of fine organization should be maintained. The outlook for frozen-dried arterial grafts is most encouraging, and it may not be too rash to say that the practical problems raised by their storage and use are now in principle solved. Bone homografts, even after freeze-drying, show high promise of surgical use. Corneal grafting, from the nature of things, can only approach asymptotically to 100 per cent success : through no fault of the surgeon, it is at present the only tissue for which storage methods are badly in arrears of methods of transplantation. Scientific workers can help tho ophthalmic surgeon by devising efficient storage methods and also by attempting to analyse the occasional failure of corneal homografts after promising beginnings: such failures may prove to be of immunological origin. 